For permission to reprint all works in this volume by each of the following poets, grateful acknowledgment is made to the holders of copyright, publishers, or representatives named below.

Giulio Einaudi Editore

Nanni Balestrini: "Senza lacrime per le rose," from Poesie practiche, 1954-1969. Franco Fortini: "Foglio di via," from Foglio di via. Alfredo Giuliani: "Resurrezione dopo la pioggia," "Il vecchio," "I giorni aggrappati alla città," "Predilezioni," "Azzuro pari venerdi,, from Povere Juliet e altre poesie; "Chi l'avrebbe detto," from Chi l'avrebbe detto. Pier Paolo Pasolini: "Fevràr," "Il di da la me muàrt," from La nuova gioventù; "Le ceneri di Gramsci," "Il pianto della scavatrice"-l, II, from Le ceneri di Gramsci.

Giangiacomo Feltrinelli Editore

Nanni Balestrini: "In questo modo," "L'istinto di conservazione," "Apologo dell'evaso," "Tape Mark," "De cultu virginis," from Come si agisce; "Ma noi facciamone un'altra," from Ma noi facciamone un'altra. Franco Fortini: "Camposanto degli inglesi," "Agro inverno," "Agli amici," "Lettera," "A Santa Croce," from Poesia ed errore. Alfredo Giuliani: "Lettera della terapia montana," "Il canto animale," from /I tautofono. Giancarlo Marmori: "Il tuo totem è la serpe opulenta," "Stava sempre medicando qualche sua umana ferita," "Ancora la tua traccia fine d'animale," "Nulla conosco del sonno che t'ammansisce," from Poesie. Elio Pagliarani: "Oggetti e argumenti per una disperazione," from Lezioni di fisica e fecaloro. Antonio Porta: "Europa cavalca un toro nero," "Dialogo con Herz," "Il vento soffia sul limite," "La pelliccia del 
castoro," from "Zero," "Aprire," from "Rapporti umani" - XII, XIII, XIV, XV, from I rapporti; "Utopia del nomade," from Week-end. Roberto Roversi:

"Giorno di mercato," "La bomba di Hiroshima," "Le costumanze politiche," "Iconografia ufficiale," from Dopo Campoformio. Edoardo Sanguineti: "Laborintus" - 1, 2, "Erotopaegnia" - 3, 4, 5, 6, 7, "Purgatorio de l'inferno" - 1, 2, 3, 14, 15, 16, 17, from Catamerone. Adriano Spatola: "Il boomerang," "Sterilità in metamorfosi," from L'oblò. Paolo Volponi: "La cometa," "Altra voce," "Stanze romane," from L'antica moneta; "La fine dell'estate," "Le mura di Urbino," "Domani è già marzo," from Le porte dell'Appennino.

\section{Garzanti Editore}

Pier Paolo Pasolini: "Il sole, il sole," "Un lungomare," from Poesia in forma di rosa; "L'anello," from Trasumanar e organizzar; Amelia Rosselli: from Variazioni belliche; "Neve," "Nessuno," "Dialogo con i morti," "Sciopero generale 1969," from Documento (1966-1973).

\section{Arnoldo Mondadori Editore}

Bartolo Cattafi: "Antracite," from Le mosche del meriggio; "Qualcosa di preciso," "Apertura d'ali," from Qualcosa di preciso; "Al quinto piano," "Tabula rasa," from L'osso, I'anima; "Filo nero," "Il buio," "Vulnerabilità," from La discesa al trono, 1972-1975. Luciano Erba: "La Grande Jeanne," "Un equazione di primo grado," "Terra e mare," "Incompatibilità," "Lombardo-veneto," "Tabula rasa?," from II male minore. Franco Fortini: "Il Comunismo," from Una volta per sempre; "Il presente," "Gli ospiti," from Questo muro. Giovanni Giudici: "Mi chiedi cosa vuol dire," "Se sia opportuno trasferirsi in campagna," "Epigramma romano," from La vita in versi. Giancarlo Majorino: "Strappo," "La miopia," "Anniversario," "Bisoccupato," "Paesaggio industriale," from Lotte secondarie. Elio Pagliarani: "Umilmente confesso che sono mortale," "Canto d'amore," "Narcissus pseudonarcissus," "Poème antipoème," from La ragazza Carla e altre poesie. Nelo Risi: "I meli i meli i meli," "L'altra faccia," from Polso teso; "Trinità dei Monti," from II contromemoriale; "Tautologia," "Manovre," from Pensieri elementari; "Il teatro privato," from "Variazioni sul bianco," from Amica mia nemica. Rocco Scotellaro: "È un ritratto tutto piedi," "Alla figlia del trainante," "Camminano sulle zampe dei gatti," "Notte in campagna," "La luna piena," "Gli abigeatari," from $\dot{E}$ fatto giorno. Andrea Zanzotto, "Declivio su Lorna," from Dietro il paesaggio; "Da un'altezza nuova," from Vocativo; "Ecloga IV," "13 settembre 1959 (variante)," from IX Ecloghe; "La perfezione della neve," "Al mondo," from La beltà; "Subnarcosi," from Pasque.

Lamberto Pignotti

from "Vita zero" - 3, 6, 8, 11, 15, 17, 22, from "Riduzioni"-IV, VII, VIII, IX, "Poesia e politica," from Nozione di uomo.

Cesare Vivaldi

"Viaggio," "Settembre," from I/ cuore di una volta; "Madre non dimentico," "A Giovanni," from Poesie liguri; "Il muro," from Dettagli. 
Some of these translations appeared for the first time in Paris Review, Chicago Review, Small Moon, Laughing Bear, Poetry Now, Chariton Review, and Italian Poetry Today: Currents and Trends, edited by Ruth Feldman and Brian Swann, and published by the New Rivers Press. 
